\title{
FÉVRIER 1959
}

\section{REVUE}

\section{INTERNATIONA LE}

\author{
DE LA
}

\section{CROIX-ROUGE}

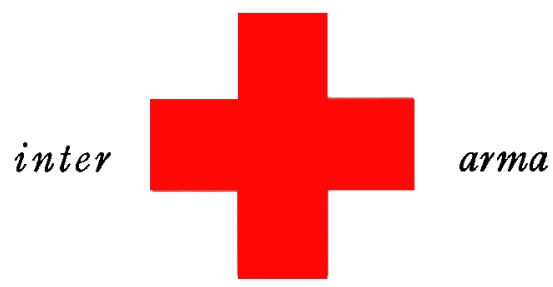

caritas

COMITE INTERNATIONAL DE LA CROIX-ROUGE G E N È V E 


\title{
COMITÉ INTERNATIONAL DE LA CROIX-ROUGE
}

\section{à $\mathrm{G}$ enève}

\author{
Président d'honneur: M. MAX HUBER, docteur en droit, \\ ancien président de la Cour permanente de justice internationale
}

MM. LEOPOLD BOISSIER, docteur en droit, professeur honoraire de l'Université de Genève, ancien secrétaire général de l'Union interparlementaire, président (1946) 1

JACQUES CHENEVIERE, docteur ès lettres h. c. (1919)

$M^{11}$ LUCIE ODIER, ex-chef du Service des infirmières-visiteuses de la Section genevoise de la CroixRouge suisse (1930)

MM. CARL J. BURCKHARDT, docteur en philosophie, ancien ministre de Suisse en France (1933)

MARTIN BODMER, docteur en philosophie h. c. (1940)

ERNEST GLOOR, docteur en médecine (1945)

PAUL RUEGGER, ancien ministre de Suisse en Italie et en Grande-Bretagne, membre de la Cour Permanente d'Arbitrage (1948)

HENRI GUISAN, général, ancien commandant en chef de l'armée suisse (1948)

RODOLFO OLGIATI, ancien directeur du Don suisse (1949)

$M^{11 \theta}$ MARGUERITE VAN BERCHEM, ancien chef de service de l'Agence centrale des prisonniers de guerre (1951)

MM. FRÉDÉRIC SIORDET, avocat, conseiller du Comité international de la Croix-Rouge de 1943 à 1951, vice-président (1951)

MARCEL JUNOD, docteur en médecine, délégué du Comité international de la Croix-Rouge de 1935 à 1946, vice-président (1952)

GUILLAUME BORDIER, ingénieur dipl. E.P.F., M.B.A. Harvard, banquier (1955)

ADOLPHE FRANCESCHETTI, docteur en médecine, professeur de clinique ophtalmologique à l'Université de Genève (1958)

HANS BACHMANN, docteur en droit, secrétaire général adjoint du Comité international de la Croix-Rouge de 1944 à 1946 (1958)

JACQUES FREYMOND, docteur ès lettres, directeur de l'Institut universitaire de hautes études internationales, professeur à l'Université de Genève (1959)

\section{Direction :}

MM. ROGER GALLOPIN, docteur en đroit, directeur exécutif

JEAN S. PICTET, docteur en droit, directeur des Affaires générales

EDOUARD de BONDELI, sous-directeur, Services financiers et administratifs

CLAUDE PILLOUD, sous-directeur, Service juridique

1 Les années indiquées dans les parenthèses désignent les dates de nomination des membres du Comité international. 


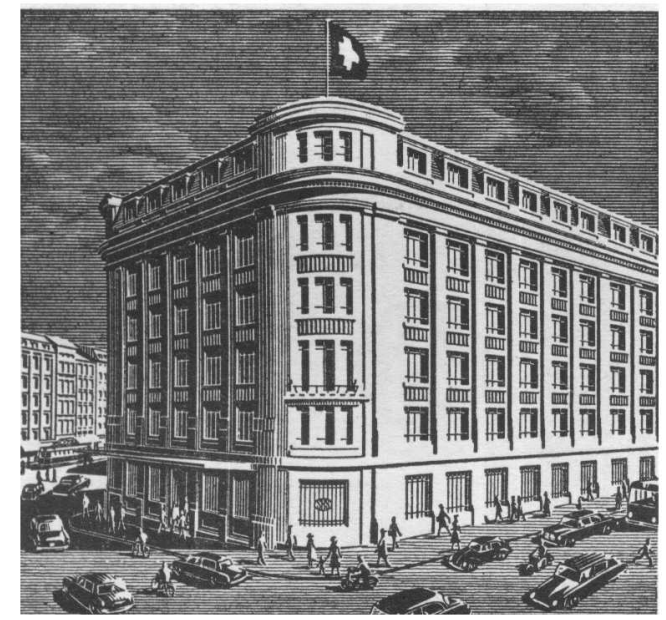

\section{SOCIETÉ DE}

\section{BANQUE SUISSE} SCHWEIZERISCHER BANKVEREIN SOCIETA DI BANCA SVIZZERA SWISS BANK CORPORATION

\section{GEN ÈVE}

BALE BIENNE LA CHAUX-DE-FONDS LAUSANNE NEUCHATEL ST-GALL SCHAFFHOUSE ZURICH LONDRES NEW-YORK 


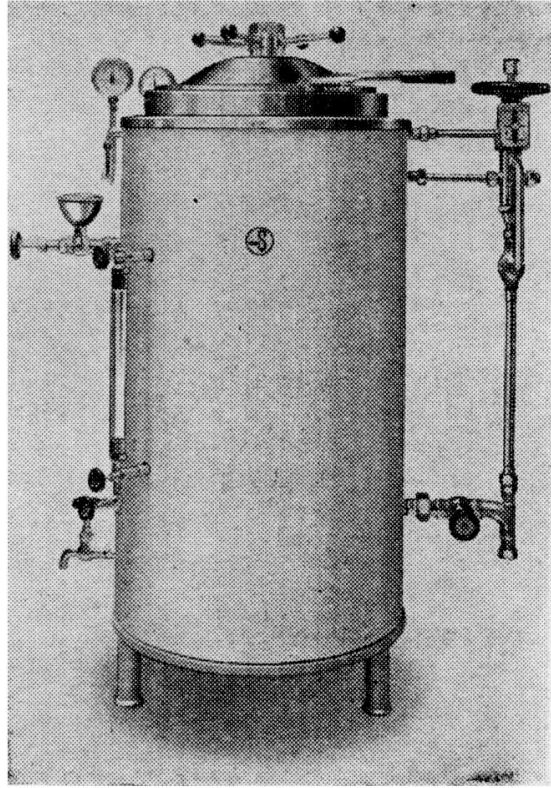

Lausanne

3, place Pépinet-(021) 228672
Basel

Streitgasse $4 \cdot(061) 248281$

\section{Malson suisse}

de réputation mondiale,

fabriquant et fournissant

les plus modernes

spécialités techniques

pour médecins ot hôpitaux

\section{SCHAERER SA.}

\section{Fabrique a WABERN pros Berne}

Case postale transit 1195 Berno

Cendve, 15, rue du Rhane - (022) 242537

Bern, Thesterplotz 4 - (031) 52926

Zürich 1, Lơwenstrasse 58 - (051) 235224

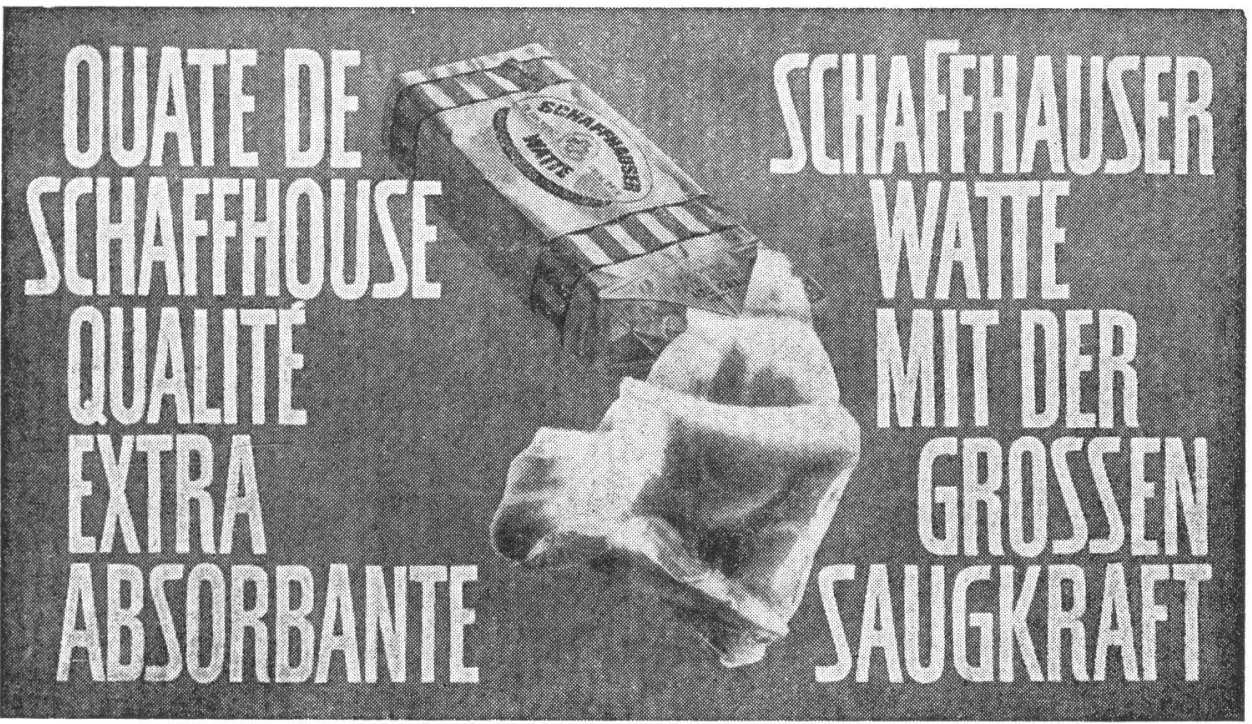




\section{REVUE INTERNATIONALE DE LA CROIX-ROUGE}

QUARANTE ET UNIËME ANNEEE - N 482

FÉVRIER 1959

\section{SOMMAIRE}

Frédéric Siordet : La leçon de Solférino . . . . . . . . . . . . . 53

G. N. Bugge : La Croix-Rouge danoise au Groenland . . . . . . . 60

\section{COMITÉ INTERNATIONAL DE LA CROIX-ROUGE}

Démission . . . . . . . . . . . . . . . . . . 67

Le CICR et le conflit algérien . . . . . . . . . . . . . . . 68

Conventions de Genève . . . . . . . . . . . . . . . . . . 73

Nouvelles d'ici et de partout . . . . . . . . . . . . . . . . 74

NOUVELLES DE SOCIÉTÉS NATIONALES

Albanie .................. . 83

Allemagne (République fédérale) . . . . . . . . . . . . 86

\section{FAITS ET DOCUMENTS}

La pénurie du personnel sanitaire . . . . . . . . . . . . . 88

\section{BIBLIOGRAPHIE}

A TRAVERS LES REVUES 


\section{SUPPLEMENTS DE LA REVUE}

EN LANGUE ANGLAISE

Claude Pilloud : The Geneva Conventions and Telegraphic communications. - News Items.

EN LANGUE ESPAGNOLE

Claude Pilloud : Los Convenios de Ginebra y las comunicaciones telegraficas. - Informaciones del mundo entero.

EN LANGUE ALLEMANDE

Claude Pilloud : Die Genfer Abkommen und die telegraphische Nachrichtenübermittlung. - Náchrichten von Nah und Fern.

LA

REVUE INTERNATIONALE DE LA CROIX-ROUGE est publiée chaque mois par le Comité international de la Croix-Rouge

7, avenue de la Paix, Genève (Suisse) - Compte de chèques postaux I. 1767

Abonnement un an : Fr. 20,-; le numéro"Fr. 2,-

Pourr les pays suivants: Algérie, Allemagne, Autriche, Belgique, Danemark, Finlande, France, Italie, Luxembourg, Maroc, Nor'ège, Pays-Bas, Portugat, Sarre. Suede, Tunisie, Vatican, on peut s'abonner auprès des bureaux de poste (Fr, s. 20,50).

Rédaction : JEAN-G. LOSSIER 